\title{
A Sequential Analysis of Early Development of Behaviors in Chicks.
}

TAKASHI TORIGOE ${ }^{1)}$

Hiroshima University

Developmental studies of behavior have examined changes in temporal patterning of behavior as well as each appearance of new behavioral pattern (2). Of the various temporal aspects of behaviors mentioned by FAGEN et al ( 3 ), the present study deals with behavioral sequences. The sequence of behaviors occurring in a social setting involves two aspects: intra- and inter-subject behavioral sequences, which are not totally separate processes but partly dependent on each other. However, only one of these processes has been analysed thus far $(\mathbf{4}, \mathbf{6}, \mathbf{9})$. In this connection VAN DEN BERCKEN and COOLS (13) developed a new sequential model which considered both aspects of sequences simultaneously. Using this model the present study attempted to describe sequential patterning of behaviors and their developmental changes in socially reared chicks.

\section{Method}

Subjects and their rearing conditions:

The subjects were 20 white leghorn chicks. Eggs were obtained from a commercial supplier and incubated in a forced draft incubator (Showa Electric Incubator P-01) maintained at $37.5^{\circ} \mathrm{C}$. Chicks were hatched in groups and reared in pairs. The home cage, in which two chicks were housed, was a wooden box $(25 \times 38 \times 38 \mathrm{~cm})$. One of its longer walls was transparent and the floor of the box was covered with sand, which was presumed to promote natural behaviors. Home cages were separated from each other. A brooder containing six such cages was heated constantly at $33^{\circ} \mathrm{C}$ and illuminated by a $100 \mathrm{w}$ fluorescent lamp for a period of about $12 \mathrm{~h}$ daily. Food and water were always available in each home cage.

Procedure:

In this study, the 0-day of age refers to the day of hatching. But in case of

1) The author thanks Prof. Yoshimasa Habu, Ph. D., Department of Psychology, Hiroshima University, for his helpful comments on the manuscript. 
hatching at night, the 0-day of age was arbitrarily defined as the first day when the chick was found to be hatched. The observation time of the day was held as close as possible to the time of hatching in order to maintain the homogeneity of subjects.

Before observation each home cage was gently transferred by the experimenter from the brooder to the observational room, where the cage was heated approximately at $33^{\circ} \mathrm{C}$ and illuminated by a $100 \mathrm{w}$ fluorescent lamp. Each pair was observed $30 \mathrm{~min}$ a day after $10 \mathrm{~min}$ adaptation at $1-, 3-, 5-$, and 7 -day of age. A video system (VTR, SL-J9; video color camera, DXC-17; Sony, Co.Ltd.) was used for recording all observations.

Analysis:

Table 1 shows the behavior categories. The intra- and inter-subject sequences

Table 1. The behavioral categories.

(1) $\operatorname{Approach}(\mathrm{Ap})$

(2) Bill to Bill(BB)

(3) Bill Open (BO)

(4) Bill Clean $(\mathrm{BC})$

(5) Body Shake(BS)

(6) Distress Call(DC)

(7) Does Nothing (DN)

(8) Drink water (Dr)

(9) Eat(E)

(10) Head Shake(HS)

(11) Head Scratch $(\mathrm{HSc})$

(12) Peck (P)

(13) Peck at Bill(PB)

(14) Peck at Eye (PE)

(15) Peck at Foot (PF)

(16) Peck at Head (PH)

(17) Peck at the other body parts(PO)

(18) $\operatorname{Preen}(\operatorname{Pr})$ :one chick pecks at the other's body parts, excluding

:one chick rushes or runs to the other (excluding incidental and slow approach).

:one chick moves its bill toward the other's, holding this position for a while without bill contact.

: the chick opens its bill for a short while.

: the bill is scraped against the cage in a side-toside motion.

:the chick shivers in a fixed manner.

: the chick peeps.

: the chick does nothing in spite of behavioral changes of the partner.

:the chick rapidly shakes its head horizontally several times in a fixed manner.

: the chick scratches its head with its foot.

:the chick pecks at the cage (excluding food and water).

:one chick pecks at the other's bill.

: one chick pecks at the other's eye.

: one chick pecks at the other's foot.

: one chick pecks at the parts of the head of the other chick, excluding (13) and (14).

(13), (14), (15) and (16). 
TORIGOE: Early Development of Behavior in Chicks

(19) $\operatorname{Push}(\mathrm{Pu})$

(20) $\operatorname{Scratch}(\mathrm{Sc})$

(21) Sleep(Sl)

(22) Self Peck(SP)

(23) Body Stretch (Str)

(24) Wing Flap(WF)

(25) Wing Up(WU) :one chick pushes the other.

:the chick scratches the floor with its feet in a fixed manner.

:the chick is inactive with eyes closed for at least several seconds.

: the chick pecks at its own body parts.

:the chick stands on one leg and stretches its body

and neck together with the wing and leg of the opposite side.

:the chick raises up both of its wings, holding this position for a while.

of these behaviors in a pair setting were recorded by a modified sequence sampling method (8). Behavioral repetition was recorded only in the case where one chick retained the same behavioral pattern in spite of behavioral changes of the partner.

Based on these records, two analyses were carried out: informational analysis and chi-square statistical analysis.

An informational analysis was performed by the same method as that of VAN DEN BERCKEN and COOLS (13). In the present study two measures, normalized partial auto-covariability and normalized partial cross-covariability, were calculated. For brevity, auto-constraint and cross-constraint are used below. These two measures show the extent to which the variability of ongoing behavior (Res) is constrained by its own previous behavior (Pre) and the other's previous behavior (Sig), respectively.

Next, intra- and inter-subject behavioral sequences were separately broken down into dyads and two transition matrices (Pre-Res, Sig-Res) were produced. A chi-square statistical analysis (4) was performed on both matrices. Two manipulations were specific to the method used in the present analysis. One was that the less frequent behaviors (occurring less than 70 times) were excluded from analysis because of the possibility that only a few exceptional subjects might have influenced the results. The other was that only the positive association of dyads was considered, because this study was mainly interested in co- or serial occurrence tendencies among behaviors rather than mutually inhibitive ones.

\section{Results}

Table 2 shows the number of behaviors sampled for each day of observation. 
According to the criterion set by FAGEN and Young (3) the sample size can be considered large enough for statistical analysis.

Table 2. The number of behaviors sampled in each day of observation.

\begin{tabular}{lcccc}
\hline Day of age & 1 & 3 & 5 & 7 \\
\hline $\begin{array}{l}\text { The number of behaviors } \\
\text { sampled }\end{array}$ & 4278 & 4518 & 5628 & 5509 \\
\hline
\end{tabular}

Figure 1 shows the values of auto- and cross-constraints over seven days after hatching, indicating that the values of cross-constraint were high at 1- and 3-day of age and decreased thereafter, and the auto-constraint increased greatly between 1- and 3-day of age.

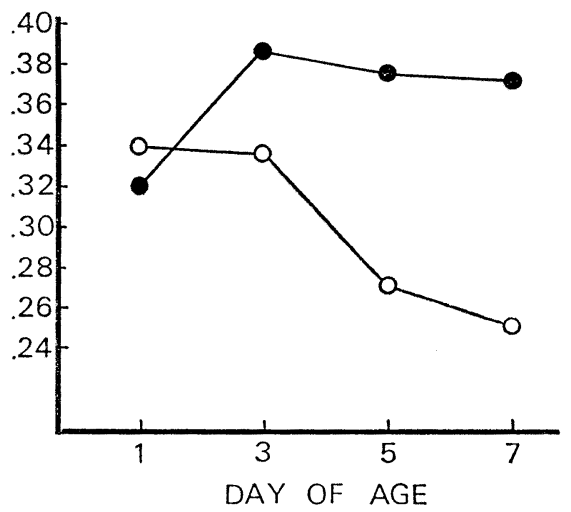

Fig. 1. The auto-constraint $(-)$ and cross-constraint $\left(\mathrm{O}_{-}-\right.$).

Figure 2 shows the system diagrams of intra- and inter-subject behavioral sequences (for brevity, Pre-Res system and Sig-Res system are used below) at 1-, $3^{-}, 5^{-}$, and 7-day of age on the basis of the chi-square statistical analysis. The arrows between the circles represent significant $(p<.05)$ positive associations between two behaviors. This figure indicates two characteristics underlying Pre-Res and Sig-Res systems.

First, the most striking characteristic of peer-interaction in newly hatched chicks was the behavioral synchrony, which was shown as a looped arrow in the Sig-Res systems. This implies that chicks match their behaviors to each other. Since 1-day of age this phenomenon was observed through various behaviors, such as $\mathrm{PB}, \mathrm{P}$, $\mathrm{E}, \mathrm{Sl}, \mathrm{Pu}, \mathrm{Pr}, \mathrm{DC}$, and Sc.

Second, social pecks were classified into three types: $\mathrm{PE}-\mathrm{PF}, \mathrm{PB}$, and $\mathrm{PO}$, on 


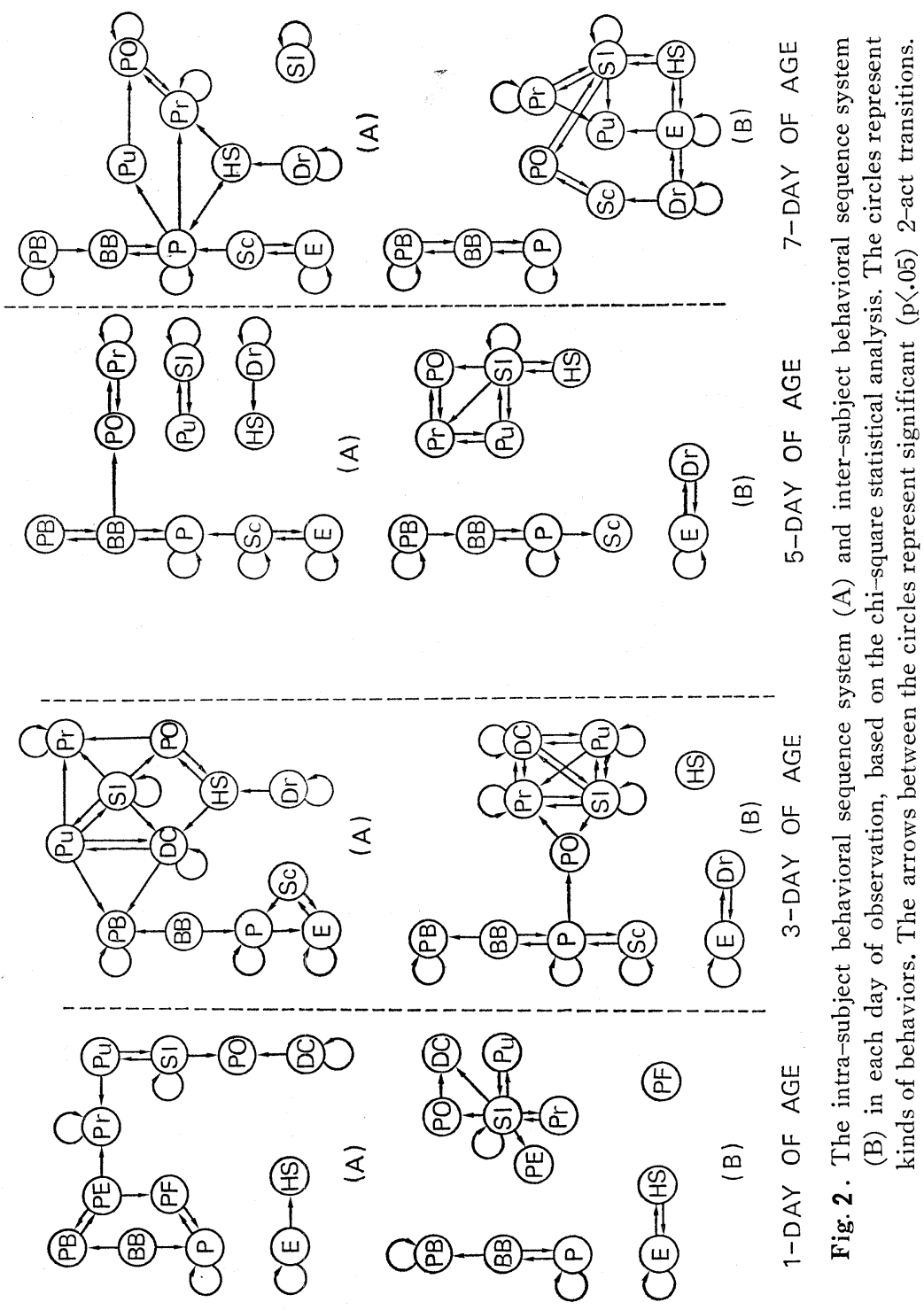


the basis of the temporal context of their occurrences and their developmental changes. $\mathrm{PE}$ and $\mathrm{PF}$ frequently occurred immediately after hatching and contingently with $\mathrm{P}$, decreasing rapidly thereafter. $\mathrm{PB}$ occurred also contingently with $\mathrm{P}$ but did not decrease over seven days after hatching. PO occurred in temporal contiguity with $\mathrm{Pr}, \mathrm{Pu}$, and/or Sl. This occurrence was quite different from other social pecks which had a close relationship with $\mathrm{P}$.

\section{Discussion}

The present study described quantitatively the temporal structure (especially serial occurrence tendency) of behaviors in chicks and its developmental changes over seven days after hatching.

An informational analysis showed the high values of cross-constraint at 1-day of age. Thereafter, the cross-constraint decreased and the auto-constraint increased. This result suggests that during the early period of peer-interaction chicks are socially sensitive and their ongoing behaviors are more determined by the other's behaviors rather than by their own previous behaviors, and that this relation reverses over time.

Social pecks could be classified into three types, PE-PF, PB, and PO, which occurred in different contexts and took different developmental courses from each other. Thus social pecks should not be counted as a single category $(\mathbf{1}, \mathbf{5}, \mathbf{7})$. Further studies are necessary to specify the function of each type of social pecks.

The behavioral synchrony, shown as a looped arrow in the Sig-Res system (Fig. 2), showed marked characteristics of peer-interaction. This was observed through various behaviors shortly after hatching. But only the synchrony of eating was investigated so far $(\mathbf{1 0}, 11,12)$. This referred to social facilitation, in which a satiated chick continued to eat at the sight of feeding chicks, and which was assumed to have an adaptive value in that it made them grow faster. It is not likely that the same mechanism which produces social facilitation underlies all the behaviors synchronized between chicks. For example, $\mathrm{PB}$ or $\mathrm{Pu}$ is not assumed to promote chicks to grow. Gallus chicks in their natural habitat live under as high a pressure of predation as other species of ground-nesting birds. So chicks are hatched precociously and their mother abandons the nest a few days after hatching. Under these circumstances, it may possibly be more adaptive for chicks to synchronize behaviorally to each other than otherwise, for the possibility for them to be detected by a potential predator may be reduced when they all rest or travel 
rapidly at the same time rather than when each chick behaves in an individual manner. VINCE (14) reported the synchronization of hatching in quail, the origin of which could be experimentally traced back to auditory communication among embryos. The present study did not control for prenatal and auditory experiences, so further studies focusing on this aspect are necessary to deal with the origin and mechanism of the behavioral synchrony.

\section{REFERENCES}

1. Evans, R. M. Early aggressive responses in domestic chicks. Animal Behaviour, 1967, 16, 24-28.

2. BEKOFF, M. The development of social interaction, play, and metacommunication in mammals: An ethological perspective. Quarterly Review of Biology, 1972, 47, 412-434.

3. FAGEN, R.M. and YounG, D.Y. Temporal patterns of behaviors: Duration, intervals, latencies, and sequences. In P.W.ColgAN(ed.) Quantitative ethology. pp. 79-114, New York: John Wiley and Sons, 1978.

4. Hazlett, B. A. and Bossert, W. H. A statistical analysis of the aggressive communication systems of some hermit crabs. Animal Behaviour, 1965, 13, 355-377.

5. Hoffman, H.S., Boskoff, K.J., Eiserer, L.A., and Klein, S. H. Isolationinduced aggression in newly hatched ducklings. Journal of Comparative and Physiological Psychology, 1975, 89, 447-456.

6. POOLE, T.B. and Fish, J. An investigation of playful behaviour in Rattus norvegicus and Mus musculus. Journal of Zoology, 1975, 175, 61-71.

7. RAJECKI, D.W., IVINS, B., and RHEIN, B. Social discrimination and aggressive pecking in domestic chicks. Journal of Comparative and Physiological Psychology, 1976, 90, 422-452.

8. SteinberG, J.B. Information theory as an ethological tool. In B. A. HAzLetT (ed.)Quantitative methods in the study of animal behavior.pp.46-74, New York: Academic Press, 1977.

9. STEINBERG, J.B. and CONANT, J.R. An informational analysis of the intermale behaviour of the grass-hopper Chortophagalviridifasciata. Animal Behaviour, 1974, 22. 612-627.

10. TOlmaN, C.W. Social facilitation of feeding behaviour in the domestic chicks. Animal Behaviour, 1964, 12, 245-251. 
11. Tolman, C.W. Social feeding in chicks. Animal Behaviour, 1967, 15, 145-148.

12. TOLMAN, C. W. The feeding behaviour of domestic chick as a function of rate of pecking by a surrogate companion. Behaviour, 1967, 29, 57-62.

13. VAN DEN BERCKEN, J.H. L. and CoOLS, A. R. Information-statistical analysis of social interaction and communication: An analysis-of-variance approach. Animal Behaviour, 1980, 28, 172-188.

14. VINCE, M. A. Embryonic communication, respiration and the synchronization of hatching. In R.A. HINDE (ed.) Bird vocalization. pp.233-260, New York: Cambridge University Press, 1969. 